\title{
Sphingosine-1-Phosphate (S1P) Is a Feasible Biomarker in Predicting the Efficacy of Polymyxin B-Immobilized Fiber Direct Hemoperfusion (PMX-DHP) in Patients with Septic Shock
}

\author{
Satoshi Inoue ${ }^{1}$, Yuichiro Sakamoto ${ }^{2}$, Hiroyuki Koami ${ }^{2}$, Kosuke Yamada C ${ }^{2}$, \\ Futoshi Nagashima ${ }^{1}$, Toru Miike ${ }^{2}$, Takashi Iwamura ${ }^{1}$ and Toru Obata ${ }^{3}$ \\ ${ }^{1}$ Division of Trauma Surgery and Surgical Critical Care, Saga University Hospital, Saga, Japan \\ ${ }^{2}$ Department of Emergency Medicine, Saga University Hospital, Saga, Japan \\ ${ }^{3}$ Department of Surgery, Shiga University of Medical Science, Shiga, Japan
}

Purpose: The aim of this study was to identify a useful biomarker to predict the efficacy of polymyxin B-immobilized fiber direct hemoperfusion (PMX-DHP) in patients with septic shock.

Methods: The 44 patients included in this study were divided into two groups. Group A had an increase in systolic blood pressure (SBP) over $30 \mathrm{mmHg}$ after PMX-DHP treatment. Group B had an increase in SBP less than $30 \mathrm{mmHg}$ after PMX-DHP treatment. We evaluated the clinical characteristics and demographics of both groups. We also assessed whether the cause of sepsis affected the efficacy of PMX-DHP and compared the prognosis of both groups. Finally, we investigated whether there were any significant differences in the levels of sepsis-related biomarkers, including sphingosine-1-phosphate (S1P), between both groups before PMX-DHP in an effort to identify a biomarker that could predict the efficacy of PMX-DHP.

Results: PMX-DHP significantly increased SBP regardless of the cause of sepsis. Although there was some tendency, PMX-DHP did not significantly improve the prognosis of effective cases in comparison with non-effective cases, probably because of the limited number of patients included. Among the sepsis-related biomarkers, only S1P values were significantly different between the two groups before PMX-DHP, and S1P levels were significantly increased after treatment in the effective cases.

Conclusion: S1P levels prior to PMX-DHP can be used to predict its efficacy. In addition, continuous monitoring of S1P levels can indicate the effectiveness of PMX-DHP in patients with septic shock.

(J Nippon Med Sch 2018; 85: 39-46)

Key words: PMX-DHP, sepsis, septic shock, biomarkers, S1P

\section{Introduction}

Despite recent advances in the understanding of the pathophysiological mechanism of sepsis and strategies for its treatment, the incidence of severe sepsis with septic shock continues to rise, and sepsis-related mortality remains high ${ }^{1}$. Both gram-negative (GNB) and grampositive (GPB) bacterial pathogens are the most common cause of sepsis, and fungal organisms are also increasing rapidly $^{2}$. Lipopolysaccharide (LPS) presents in the outer membrane of gram-negative bacteria. The endotoxin, the lipid A part of LPS, is another cause for sepsis and septic shock $^{3}$. In sepsis, pro-inflammatory and antiinflammatory responses coexist leading to immunosuppression and the host's erratic inflammatory response to the infection, which results in organ dysfunction. The early identification of sepsis, cultures, source control, and application of appropriate antibiotics are crucial for a better outcome in patients.

In Japan, a number of reports regarding the efficacy of direct hemoperfusion with polymyxin B-immobilized fiber (PMX-DHP) in patients with septic shock have demonstrated improved clinical outcomes including an in-

Correspondence to Satoshi Inoue, Division of Trauma Surgery and Surgical Critical Care, Saga University Hospital, 5-1-1 Nabeshima, Saga City 849-8501, Japan

E-mail: satoshimeister@gmail.com

Journal Website (http://www2.nms.ac.jp/jnms/) 
crease in systolic blood pressure ${ }^{6,7}$. Similar promising results have also been reported in Europe as well ${ }^{8}$. A change in biomarkers after PMX-DHP is reported to be useful to predict patient prognosis ${ }^{9}$.

There are several important biomarkers known to be involved in sepsis. High Mobility Group Box 1 (HMGB1), which is secreted from macrophages and monocytes, mediates inflammatory cytokines ${ }^{10}$. It is also known to trigger disseminated intravascular coagulation (DIC) and sepsis by deterioration of the coagulation system ${ }^{11}$. Other biomarkers include: Anandamide (N-arachidonoylethanolamine, AEA) and 2-arachidonoylglycerol (2-AG), endogenous agonists of cannabinoid receptors that are reported to be increased in patients with septic shock ${ }^{12}$; The plasminogen activator inhibitor-1 (PAI-1), the principal inhibitor of tissue plasminogen activator (tPA) and urokinase (uPA), which regulates the plasminogen activator to initiate fibrinolysis ${ }^{13,14}$; and Protein C (PC) which regulates blood clot size and prevents the downstream coagulation pathway through the proteolytic inactivation of the procoagulant co-factors Vllla and Va ${ }^{15}$. PAI-1 and PC activity can be a good predictor to evaluate the severity of sepsis and ongoing severe coagulopathy ${ }^{16,17}$. Further, Interleukin-6 (IL-6) is one of the most common proinflammatory cytokines and uncontrolled activation of this cytokine is an essential contributor to the pathogenesis of septic DIC ${ }^{18}$.

Of particular interest to this study, sphingosine-1phosphate (S1P), one of the sphingolipid metabolites, is a lipid mediator that regulates a wide variety of developmental and pathophysiological systems and is involved in vascular, nervous, and immune system development ${ }^{19}$. S1P also initiates intracellular signal transduction to regulate cell growth, migration, adhesion, and survival by interaction with five G-protein coupled receptors ${ }^{20}$. Since S1 $\mathrm{P}$ maintains vascular integrity and controls cytokine secretion, extracellular S1P level is related to sepsis severity $^{21}$. Therefore, S1P has been gaining increasing attention from researchers as a potential therapeutic or diagnostic target in sepsis.

It has been reported that PMX-DHP decreased levels of endocannabinoids, HMGB1, and the inflammatory cytokines $^{22-24}$ mentioned above. However, the varied responses to treatment and the mechanisms as to how PMX-DHP improves sepsis still need to be elucidated ${ }^{25}$. Moreover, most biomarkers were measured after PMX$\mathrm{DHP}^{9}$ to evaluate its efficacy. Therefore, it is imperative to find a biomarker to assess each patient prior to PMXDHP therapy to determine if it is a feasible modality to treat his or her septic shock.

Since there are no standard guidelines regarding the indication of PMX-DHP for treatment of patients with sepsis, the goal of this study was to identify a useful marker to predict the efficacy of PMX-DHP on patients with septic shock.

\section{Materials and Methods}

The present study has been approved by the institutional review board, and informed consent from all patients was obtained prior to their inclusion in this study. In this retrospective study, 44 patients with septic shock were included. All patients presented with persistent hypotension that required vasopressors to maintain their mean arterial pressure (MAP) greater than $65 \mathrm{mmHg}$. Patients underwent PMX-DHP within 24 hours from admission and were divided into two groups. Group A included patients with an increased systolic blood pressure (SBP) greater than $30 \mathrm{mmHg}$ after PMX-DHP treatment while group B included patients without an increase in SBP by PMX-DHP. A double-lumen catheter was inserted by the Seldinger technique into the femoral vein to establish venous access for hemoperfusion. PMX-DHP was run for 2 hours at a flow rate of $80 \mathrm{~mL} / \mathrm{min}$, and it was performed twice consecutively. An anticoagulant, Nafamostat mesylate (Torii Co., Ltd, Tokyo, Japan), was used during PMX-DHP.

First, clinical characteristics and demographics including sex, age, (Acute Physiology and Chronic Health Evaluation II) APACHE II scores, (Sequential Organ Failure Assessment score) SOFA scores, and DIC scores of all of the patients in both groups were analyzed.

Next, we evaluated whether PMX-DHP improved the SBP of the patients with sepsis; the causes of sepsis varied (surgical or non-surgical; gram-positive or gramnegative bacteria). The surgical group included patients with sepsis, such as peritonitis, who required surgical treatment. The patients with sepsis in the non-surgical group included patients who did not undergo surgery to control the cause of sepsis, such as those with pneumonia. The prognosis of patients with septic shock in both the A and B groups who underwent PMX-DHP was also analyzed.

Furthermore, we evaluated the level of sepsis-related biomarkers such as HMGB1, IL-6, AEA, 2-AG, PAI-1, protein $\mathrm{C}$, and antithrombin-III (AT-III) before PMX-DHP.

Finally, we evaluated whether S1P, the lipid product of sphingosine kinases (SKs) that plays an essential role in inflammatory signaling processes as well as in disease 
development and progression ${ }^{26}$, can be used as an effective predictive factor of the success of PMX-DHP on patients with sepsis in terms of an increase in SBP. S1P levels were measured before and after PMX-DHP therapy in both groups. The correlations between S1P levels in both groups before and after treatment were analyzed.

S1P, endocannabinoids and F2-isoprostane were detected using a liquid chromatography tandem mass spectrometry system (Q-trap; Applied Biosystems, Foster City, CA, USA) with the isotope dilution method. The HMGB1, IL-6, and PAI-1 levels were measured using an enzyme-linked immunosorbent assay (ELISA) (Shino-Test Corporation, Japan).

\section{Statistical Analysis}

The results were expressed as the mean \pm SD. Differences in each value were analyzed by the Wilcoxon generalized test or the chi-squared test, and the KaplanMeier survival curves were compared using a log-rank test. A p-value less than 0.05 was interpreted to be statistically significant. IBM SPSS Statistics version 22 (IBM Corp., Armonk, NY, USA) was used for statistical analyses.

\section{Results}

There were 25 cases and 19 cases in groups A and B, respectively, and each group had a variety of underlying causes of sepsis, as shown in Table 1. Peritonitis (18 cases) was the leading cause of sepsis, followed by pneumonia (13 cases).

Table 1 Underlying diseases

\begin{tabular}{lc}
\hline Underlying disease & The number of cases \\
\hline Peritonitis & $18(41.0 \%)$ \\
Pneumonia & $13(29.5 \%)$ \\
Pyothorax & $4(9 \%)$ \\
Gas gangrene & $2(4.5 \%)$ \\
Meningitis & $1(2.3 \%)$ \\
Others & $6(13.6 \%)$ \\
\hline
\end{tabular}

Patients' characteristics are shown in Table 2 and there were no statistically significant differences in their background (sex, age, APACHE II score, SOFA score, DIC score, and SBP before PMX-DHP). Notably, the APACHE II scores and SOFA scores of both groups did not show any differences, suggesting that there were no significant differences in terms of severity of their illness (Table 2).

Next, we evaluated whether the SBP was improved in surgical and non-surgical septic cases. PMX-DHP significantly improved SBP in both surgical and non-surgical patients. Intriguingly, the $\mathrm{p}$ value for surgical cases was a hundred times lower than that of non-surgical cases (Fig. 1). It is interesting to note that PMX-DHP improved the SBP of patients with septic shock regardless of the causative organism (gram-negative or positive bacteria) and PMX-DHP was significantly more effective in increasing SBP in patients with a GNB infection (Table 3). There were no statistical significances between patients' survival rate and all of the sepsis biomarkers, including S1P after PMX-DHP (data not shown). Although patients with an increased SBP after therapy tended to have a better clinical outcome, PMX-DHP did not significantly improve the prognosis of effective cases (i.e. group A that had an increased SBP greater than $30 \mathrm{mmHg}$ ) as compared to non-effective cases (i.e. group B without an increase in SBP) (Fig. 2).

In an attempt to evaluate whether there is an efficacy predictor of PMX-DHP therapy for patients with sepsis, we compared values of sepsis-related biomarkers, including IL-6, HMGB-1, AEA, 2-AG, S1P, PAI-1, and protein C, between group $\mathrm{A}$ and group $\mathrm{B}$ before PMX-DHP therapy. Table 4 shows that while other biomarkers for septic DIC did not show any significant difference between the 2 groups, the values of S1P were significantly lower in group A (group A: $71.0 \pm 110.0 \mathrm{ng} / \mathrm{mL}$ vs group B: 174.1 $\pm 167.8 \mathrm{ng} / \mathrm{mL}, \mathrm{p}=0.0193)$. Furthermore the differences in S1P level before and after PMX-DHP were analyzed between the two groups (delta S1P was obtained by sub-

Table 2 Characteristics of the patients and disease severity (effective cases vs noneffective cases)

\begin{tabular}{lccc}
\hline \multicolumn{1}{c}{ Characteristics } & Effective cases & Non-effective cases & $\mathrm{p}$ value \\
\hline Number of patients & 25 & 19 & \\
Sex $($ male/female) & $14 / 11$ & $15 / 4$ & $\mathrm{NS}$ \\
Age $($ mean \pm SD) & $61.3 \pm 14.4$ & $61.5 \pm 12.4$ & $\mathrm{NS}$ \\
APACHE II score $($ mean \pm SD) & $27.3 \pm 8.2$ & $26.7 \pm 9.3$ & $\mathrm{NS}$ \\
SOFA score (mean \pm SD) & $11.6 \pm 4.6$ & $11.6 \pm 4.8$ & $\mathrm{NS}$ \\
DIC score (mean \pm SD) & $5.2 \pm 1.9$ & $4.2 \pm 2.0$ & $\mathrm{NS}$ \\
SBP before PMX-DHP $($ mean \pm SD) & $95.1 \pm 15.7$ & $104.9 \pm 23.9$ & $\mathrm{NS}$ \\
\hline
\end{tabular}




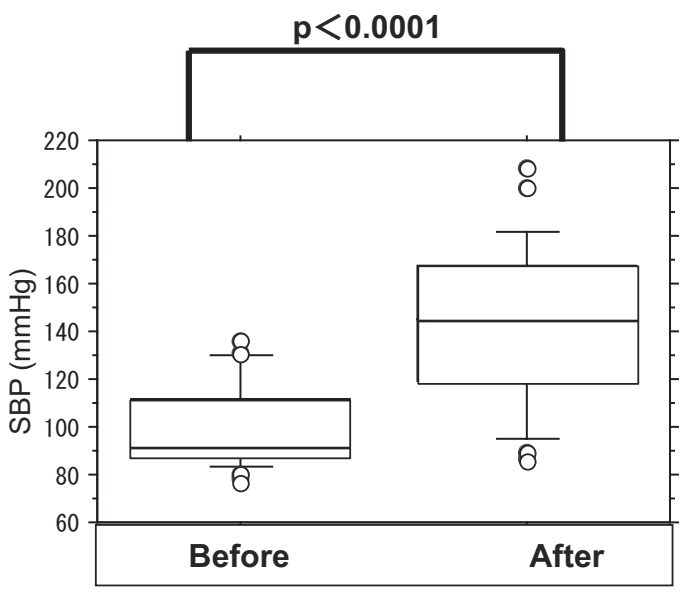

Surgical cases



Non-surgical cases

Fig. 1 The change in systolic blood pressure after polymyxin B-immobilized fiber direct hemoperfusion (PMX-DHP) based on surgical or non-surgical causation.

PMX-DHP significantly improved systolic blood pressure in both surgical and non-surgical patients.

Table 3 Increase in systolic blood pressure (SBP) by polymyxin B-immobilized fiber direct hemoperfusion (PMX-DHP) based on causative organisms

\begin{tabular}{cccc}
\hline Causative organism & $\begin{array}{c}\text { Increase in SBP } \\
\text { (greater than } 30 \mathrm{mmHg} \text { ) }\end{array}$ & $\begin{array}{c}\text { No increase in SBP } \\
\text { (greater than } 30 \mathrm{mmHg} \text { ) }\end{array}$ & p value \\
\hline Gram positive bacteria & 5 & 8 & 0.043 \\
Gram negative bacteria & 22 & 9 & \\
\hline
\end{tabular}

tracting S1P levels after PMX-DHP from the levels before treatment). It is notable that S1P levels were significantly increased in Group A that had a significant increase in SBP after PMX-DHP therapy (Fig. 3).

\section{Discussion}

The change in the hemodynamics and the conditions of a patient during severe sepsis are very dynamic and complex, which makes adjunct therapy for sepsis, other than controlling the causative lesion, greatly variable. Since sepsis tends to involve deterioration of multiple organs and coagulation systems, its treatment is very complicated and the strategy varies depending on each institution $^{27}$. The mortality of patients with severe sepsis in the first 24 hours after admission to the intensive care unit (ICU) is $30-40 \%$ before ICU discharge ${ }^{17}$. Furthermore, almost half of these patients that are diagnosed with sepsis within 24 hours of their admission die during their hospital stay ${ }^{28,29}$. Therefore, in patients with sepsis, early diagnosis and immediate management largely contribute to their outcome. Even though compliance of Early Goal Directed Therapy (EGDT) and guidelines based on the Sur- viving Sepsis Campaign is still low, it remarkably improves the prognosis of patients with severe sepsis if appropriately followed ${ }^{30,31}$.

PMX-DHP was developed and advanced in Japan. It has been utilized to successfully control endotoxemia in patients with septic shock ${ }^{6}$. Although PMX-DHP was not included in EGDT due to limited evidence of its efficacy at the time, some previous studies have shown that PMX-DHP significantly improved mortality of patients with severe sepsis due to intra-abdominal gram-negative infections with increases in MAP, decreases in vasopressor requirement, improved $\mathrm{P} / \mathrm{F}$ ratios, and improved SOFA scores when compared to the conventional therapy group $^{32,33}$. Sepsis is often caused by an unwanted complication during the course of surgical or non-surgical patients who were treated for other critical illnesses ${ }^{17,34}$. Our data showed that PMX-DHP was effective in both surgical and non-surgical cases. These findings suggest that even though source control is essential for sepsis treatment especially in patients who require surgery, supplemental therapy like PMX-DHP may be able to expedite improvement of severe sepsis conditions. 


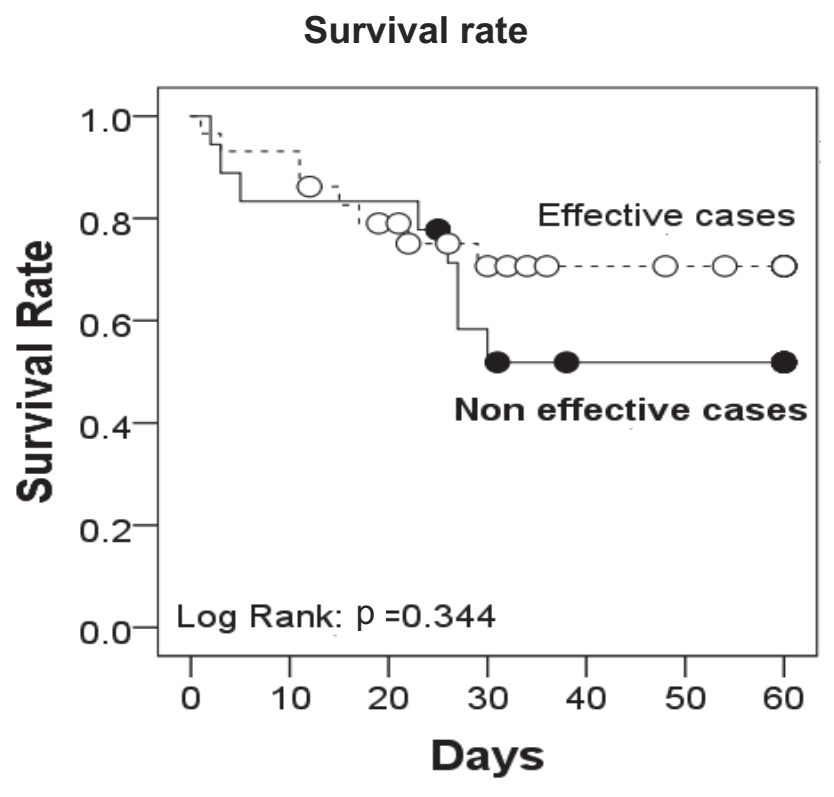

Fig. 2 Effective cases vs Non effective cases (more than 30 $\mathrm{mmHg}$ SBP increase)

The comparison of survival rate between effective (group A: an increase in systolic blood pressure (SBP) greater than $30 \mathrm{mmHg}$ ) and non-effective cases (group B: an increase in SBP less than $30 \mathrm{mmHg}$ ) after polymyxin B-immobilized fiber direct hemoperfusion (PMX-DHP). Although effective cases tended to appear to have a better prognosis, PMX-DHP did not significantly improve the prognosis of effective cases as compared to non-effective cases.

Although group A tended to have a better prognosis than group $\mathrm{B}$, unlike a previous report $\mathrm{t}^{33}$, our data did not show statistically significant correlation between the patients' prognosis and an efficacy of PMX-DHP in terms of an increase in SBP, possibly due to the low number of patients included in this study.

In the past PMX-DHP was said to only be effective against bacteremia caused by GNB, because PMX-DHP was originally designed to adsorb endotoxin to treat patients with septic shock. However, more recent studies support that it also has a beneficial effect on GPB through removal of activated monocytes and neutrophils $^{35}$. In fact, our data showed PMX-DHP increased SBP in patients with sepsis caused by both GPB and GNB, although it was significantly more effective in patients with a GNB infection. Our data suggest that PMX-DHP improves patients' hemodynamic status not only through endotoxin adsorption but also through improvement of aberrant inflammatory cytokines and mediators. Some other previous studies have also shown that PMX-DHP was effective in the reduction of endocannabinoids, high mobility group box protein 1 (HMGB1), and inflamma- tory cytokines ${ }^{6,24,36}$. Thus, PMX-DHP has been reported to improve such biomarkers involving progression of severe sepsis. However, the efficacy of PMX-DHP on patients with sepsis varies. Therefore, it is imperative to find a useful marker to predict the efficacy of the therapy.

Various factors contribute to the severity of sepsis, and $\mathrm{S} 1 \mathrm{P}$ is one of the best potential target molecules to treat sepsis because this biologically active metabolite of plasma-membrane sphingolipids has a wide range of functions that are related to sepsis onset and progression. The plasma concentrations of apoM (apolipoprotein M), a carrier for S1P, decrease dramatically during sepsis reflecting the severity of the disease ${ }^{37}$ and there is a positive correlation between plasma apoM and S1P $\mathrm{P}^{38}$. S1P levels are also significantly decreased in patients with sepsis and are inversely associated with disease severity ${ }^{21}$. Further, its value represents an aberrant inflammatory reaction in sepsis ${ }^{39}$. Since S1P protects endothelium resulting in prevention of vascular leakage and regulates vascular integrity ${ }^{40,41}$, low S1P levels may cause capillary leakage and deterioration of tissue perfusion leading to organ failure in severe sepsis. Furthermore, low S1P levels are related to LPS induced severe lung injury ${ }^{42}$. Therefore, it is reasonable to focus on the plasma S1P level to evaluate the efficacy of sepsis treatment. Our data revealed that S1P levels were significantly lower in patients in Group A with increased SBP after PMX-DHP prior to therapy, and interestingly they were markedly increased after therapy as compared to patients without an increase in SBP after therapy. These findings imply that PMX-DHP increased the SBP of patients in Group A with severe sepsis by reducing sepsis-related chemical mediators and was also able to increase S1P after therapy. We do not know whether the effect of PMX-DHP on S1P is direct or indirect. Inconsistent with previous studies ${ }^{21,37,42}$, there were no significant differences in the severity of sepsis between group A and group B in the present study despite the fact that S1P levels were significantly lower in group A than in group B. It might be possible to say that S1P can be more sensitive in reflecting the severity of sepsis than other scores such as APACHE II and SOFA. Otherwise S1P can be a very sensitive predictor for therapeutic efficacy of PMX-DHP regardless of the clinical condition of patients with sepsis. Furthermore, S1P level is known to be regulated by its synthesis by sphingosine kinases and degradation mediated by mediators including S1P phosphatases, lipid phosphate phosphatases, and S1P lyase ${ }^{43}$. Indeed, Hemdan et al. reported that administration of 4-deoxypyridoxine, an inhibitor of S1P-lyase, 
Table 4 The value of sepsis related biomarkers before polymyxin B-immobilized fiber direct hemoperfusion (PMX-DHP) (effective cases vs non-effective cases)

\begin{tabular}{lccc}
\hline \multicolumn{1}{c}{ Characteristics } & Effective cases & Non-effective cases & p value \\
\hline IL-6 $(\mathrm{pg} / \mathrm{mL})$ & $29,766.5 \pm 41,769.3$ & $18,992.4 \pm 38,071.2$ & NS \\
HMGB-1 $(\mathrm{ng} / \mathrm{mL})$ & $15.3 \pm 17.1$ & $6.9 \pm 10.4$ & NS \\
AEA $(\mathrm{ng} / \mathrm{mL})$ & $664.2 \pm 797.5$ & $414.6 \pm 272.0$ & NS \\
2AG $(\mathrm{ng} / \mathrm{mL})$ & $17.2 \pm 31.5$ & $9.1 \pm 8.9$ & NS \\
S1P $(\mathrm{ng} / \mathrm{mL})$ & $71.0 \pm 110.0$ & $174.1 \pm 167.8$ & 0.0193 \\
PAI-1 $(\mathrm{ng} / \mathrm{mL})$ & $159.5 \pm 54.5$ & $135.0 \pm 69.8$ & NS \\
Protein C $(\%)$ & $31.3 \pm 10.7$ & $46.9 \pm 38.8$ & NS \\
\hline
\end{tabular}

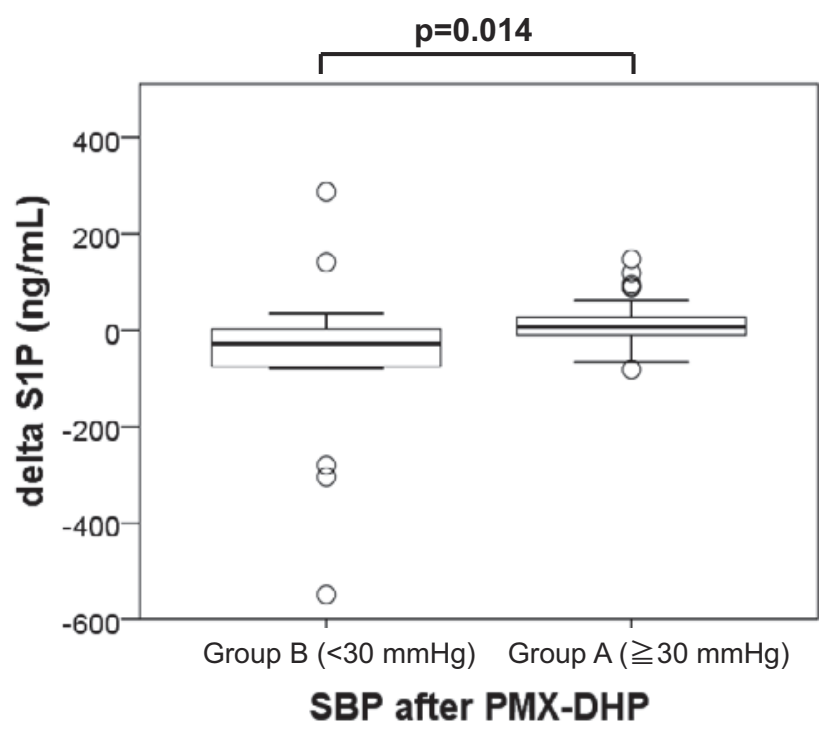

Fig. 3 Change in S1P level after the PMX-DHP in patients with or without increase in SBP

The change in sphingosine-1-phosphate (S1P) level after polymyxin B-immobilized fiber direct hemoperfusion (PMX-DHP) in patients with or without increased systolic blood pressure (SBP). The delta S1P was obtained by subtracting S1P levels after the PMX-DHP from S1P values before treatment. The delta S1P was significantly higher in patients with an increased SBP after PMX-DHP, which means that PMX-DHP increased the S1P value significantly more in effective cases as compared to ineffective cases.

improved recovery from sepsis in mice models ${ }^{44}$. Thus, PMX-DHP might be able to capture inhibitory mediators of S1P such as S1P lyase resulting in an increase in S1P level and improvement of SBP. However, detailed investigations are warranted to elucidate the detailed mechanism of this phenomenon.

The present study has some limitations to be noted. The study was performed in a single institution with a small sample size. Since patients had various etiological backgrounds, discrepancy could exist in each group.
Moreover, the efficacy of PMX-DHP might be affected by prior hemodynamic status. Fluid volume, dose of catecholamine given, and timing depended on each physician's decision, so each patient had a different hemodynamic condition before initiating PMX-DHP.

Thus, further studies with increased sample size, appropriate adjustment of patients' background, and robust institutional protocol for PMX-DHP are required to improve the limitations of the current study.

In the present study, we evaluated biomarkers that are relevant to the status of sepsis. Our data analysis revealed that S1P was the only marker included in this study that could predict significant efficacy of PMX-DHP. Moreover, the present study also showed that S1P levels were increased when PMX-DHP improved patients' SBP. Therefore, S1P may be useful to monitor the efficacy of PMX-DHP as well. Our data showed for the first time that low plasma S1P level in patients with sepsis could be a very useful predictor for successful PMX-DHP therapy.

\section{Conclusion}

Although PMX-DHP has limited evidence to date for sepsis treatment, several positive results have been shown. Due to its pathophysiological complexity, it is very difficult to evaluate the efficacy of adjunctive treatment for sepsis. Our data suggests that S1P can be a useful biomarker to predict the efficacy of PMX-DHP for patients with septic shock.

Acknowledgement: We would like to thank Dr. Janet Markman for editing the manuscript.

Conflict of Interest: The authors declare no conflict of interest. 


\section{References}

1. Hall MJ, Williams SN, DeFrances CJ, Golosinskiy A: Inpatient care for septicemia or sepsis: a challenge for patients and hospitals. NCHS Data Brief 2011; 1-8.

2. Martin GS: Sepsis, severe sepsis and septic shock: changes in incidence, pathogens and outcomes. Expert Rev Anti Infect Ther 2012; 10: 701-706.

3. Yao Z, Foster PA, Gross GJ: Monophosphoryl lipid A protects against endotoxic shock via inhibiting neutrophil infiltration and preventing disseminated intravascular $\mathrm{CO}^{-}$ agulation. Circ Shock 1994; 43: 107-114.

4. Vincent JL, Opal SM, Marshall JC, Tracey KJ: Sepsis definitions: time for change. Lancet 2013; 381: 774-775.

5. Park SK, Shin SR, Hur M, Kim WH, Oh EA, Lee SH: The effect of early goal-directed therapy for treatment of severe sepsis or septic shock: A systemic review and metaanalysis. J Crit Care 2017; 38: 115-122.

6. Sakamoto $\mathrm{Y}$, Mashiko $\mathrm{K}$, Obata T, Matsumoto H, Hara $\mathrm{Y}$, Kutsukata N, Yamamoto Y: Clinical responses and improvement of some laboratory parameters following polymyxin B-immobilized fiber treatment in septic shock. ASAIO J 2007; 53: 646-650.

7. Tsushima K, Kubo K, Koizumi T, Yamamoto H, Fujimoto K, Hora K, Kan-Nou Y: Direct hemoperfusion using a polymyxin B immobilized column improves acute respiratory distress syndrome. J Clin Apher 2002; 17: 97-102.

8. Perego AF, Morabito S, Graziani G, Casella GP, Parodi O: [Polymyxin-B direct hemoperfusion (PMX-DHP) in gram negative sepsis]. G Ital Nefrol 2006; 23 (Suppl 36): S94102.

9. Ueno $\mathrm{T}$, Ikeda $\mathrm{T}$, Ikeda $\mathrm{K}$, Taniuchi $\mathrm{H}$, Suda $\mathrm{S}$, Yeung MY, Matsuno N: HMGB-1 as a useful prognostic biomarker in sepsis-induced organ failure in patients undergoing PMXDHP. J Surg Res 2011; 171: 183-190.

10. Wang $\mathrm{H}$, Bloom $\mathrm{O}$, Zhang $\mathrm{M}$, Vishnubhakat JM, Ombrellino $\mathrm{M}$, Che J, Frazier A, Yang H, Ivanova S, Borovikova L, Manogue KR, Faist E, Abraham E, Andersson J, Andersson U, Molina PE, Abumrad NN, Sama A, Tracey KJ: HMG-1 as a late mediator of endotoxin lethality in mice. Science 1999; 285: 248-251.

11. Yamato M, Minematsu Y, Fujii J, Mori K, Minato T, Miyagawa S, Fujimura R, Morikage N, Arata $Y$, Nakano C, Wada A, Ito T: Effective combination therapy of polymyxin-B direct hemoperfusion and recombinant thrombomodulin for septic shock accompanied by disseminated intravascular coagulation: a historical controlled trial. Ther Apher Dial 2013; 17: 472-476.

12. Maccarrone M, Bari M, Battista N, Finazzi-Agro A: Endocannabinoid degradation, endotoxic shock and inflammation. Curr Drug Targets Inflamm Allergy 2002; 1: 53-63.

13. Sakata Y, Murakami T, Noro A, Mori K, Matsuda M: The specific activity of plasminogen activator inhibitor-1 in disseminated intravascular coagulation with acute promyelocytic leukemia. Blood 1991; 77: 1949-1957.

14. Sprengers ED, Akkerman JW, Jansen BG: Blood platelet plasminogen activator inhibitor: two different pools of endothelial cell type plasminogen activator inhibitor in human blood. Thromb Haemost 1986; 55: 325-329.

15. Marlar RA: Protein $C$ in thromboembolic disease. Semin Thromb Hemost 1985; 11: 387-393.

16. Koyama K, Madoiwa S, Nunomiya S, Koinuma T, Wada M, Sakata A, Ohmori T, Mimuro J, Sakata Y: Combination of thrombin-antithrombin complex, plasminogen activator inhibitor-1, and protein $C$ activity for early identification of severe coagulopathy in initial phase of sepsis: a prospective observational study. Crit Care 2014; 18: R13.

17. Ishikura $H$, Nishida $T$, Murai $A$, Nakamura $Y$, Irie $Y$, Tanaka J, Umemura T: New diagnostic strategy for sepsisinduced disseminated intravascular coagulation: a prospective single-center observational study. Crit Care 2014; 18: R19.

18. Rittirsch D, Flierl MA, Ward PA: Harmful molecular mechanisms in sepsis. Nat Rev Immunol 2008; 8: 776-787.

19. Proia RL, Hla T: Emerging biology of sphingosine-1phosphate: its role in pathogenesis and therapy. J Clin Invest 2015; 125: 1379-1387.

20. Blaho VA, Hla T: An update on the biology of sphingosine 1-phosphate receptors. J Lipid Res 2014; 55: 15961608.

21. Winkler MS, Nierhaus A, Holzmann M, Mudersbach E, Bauer A, Robbe L, Zahrte C, Geffken M, Peine S, Schwedhelm E, Daum G, Kluge S, Zoellner C: Decreased serum concentrations of sphingosine-1-phosphate in sepsis. Crit Care 2015; 19: 372.

22. Ikeda T, Ikeda K, Nagura M, Taniuchi H, Matsushita M, Kiuchi S, Kuroki Y, Suzuki K, Matsuno N: Clinical evaluation of PMX-DHP for hypercytokinemia caused by septic multiple organ failure. Ther Apher Dial 2004; 8: 293-298.

23. Sakamoto Y, Mashiko K, Matsumoto H, Hara Y, Kutsukata N, Yamamoto Y: Relationship between effect of polymyxin B-immobilized fiber and high-mobility group box1 protein in septic shock patients. ASAIO J 2007; 53: 324328.

24. Tani T, Hanasawa $K$, Kodama M, Imaizumi $H$, Yonekawa M, Saito M, Ikeda T, Yagi Y, Takayama K, Amano I, Shimaoka H, Ohta M, Okahisa T, Koga N, Fujita N, Yamasa $\mathrm{H}$ : Correlation between plasma endotoxin, plasma cytokines, and plasminogen activator inhibitor-1 activities in septic patients. World J Surg 2001; 25: 660668.

25. Salomao R, Brunialti MK, Rapozo MM, Baggio-Zappia GL, Galanos C, Freudenberg M: Bacterial sensing, cell signaling, and modulation of the immune response during sepsis. Shock 2012; 38: 227-242.

26. Snider AJ: Sphingosine kinase and sphingosine-1phosphate: regulators in autoimmune and inflammatory disease. Int J Clin Rheumtol 2013; 8.

27. Remick DG: Pathophysiology of sepsis. Am J Pathol 2007; 170: 1435-1444.

28. Padkin A, Goldfrad C, Brady AR, Young D, Black N, Rowan K: Epidemiology of severe sepsis occurring in the first $24 \mathrm{hrs}$ in intensive care units in England, Wales, and Northern Ireland. Crit Care Med 2003; 31: 2332-2338.

29. Vincent JL, Sakr Y, Sprung CL, Ranieri VM, Reinhart K, Gerlach H, Moreno R, Carlet J, Le Gall JR, Payen D, Sepsis Occurrence in Acutely Ill Patients Investigators: Sepsis in European intensive care units: results of the SOAP study. Crit Care Med 2006; 34: 344-353.

30. Barochia AV, Cui X, Eichacker PQ: The Surviving Sepsis Campaign's Revised Sepsis Bundles. Curr Infect Dis Rep 2013; 15: 385-393.

31. Fujishima S, Gando S, Saitoh D, Mayumi T, Kushimoto S, Shiraishi SI, Ogura H, Takuma K, Kotani J, Ikeda H, Yamashita N, Suzuki K, Tsuruta R, Takeyama N, Araki T, Suzuki Y, Miki Y, Yamaguchi Y, Aikawa N, Japanese Association for Acute Medicine Sepsis Registry Study G: A multicenter, prospective evaluation of quality of care and mortality in Japan based on the Surviving Sepsis Campaign guidelines. J Infect Chemother 2014; 20: 115-120.

32. Cruz DN, Perazella MA, Bellomo R, de Cal M, Polanco 
N, Corradi V, Lentini P, Nalesso F, Ueno T, Ranieri VM, Ronco C: Effectiveness of polymyxin B-immobilized fiber column in sepsis: a systematic review. Crit Care 2007; 11: R47.

33. Cruz DN, Antonelli M, Fumagalli R, Foltran F, Brienza N, Donati A, Malcangi V, Petrini F, Volta G, Bobbio Pallavicini FM, Rottoli F, Giunta F, Ronco C: Early use of polymyxin B hemoperfusion in abdominal septic shock: the EUPHAS randomized controlled trial. JAMA 2009; 301: 2445-2452.

34. Moore LJ, Moore FA, Todd SR, Jones SL, Turner KL, Bass BL: Sepsis in general surgery: the 2005-2007 national surgical quality improvement program perspective. Arch Surg 2010; 145: 695-700.

35. Miyamoto K, Kawazoe Y, Kato S: Prolonged direct hemoperfusion using a polymyxin B immobilized fiber cartridge provides sustained circulatory stabilization in patients with septic shock: a retrospective observational before-after study. J Intensive Care 2017; 5: 19.

36. Kohro S, Imaizumi H, Yamakage M, Masuda Y, Namiki A, Asai Y, Maruyama I: Anandamide absorption by direct hemoperfusion with polymixin B-immobilized fiber improves the prognosis and organ failure assessment score in patients with sepsis. J Anesth 2006; 20: 11-16.

37. Kumaraswamy SB, Linder A, Akesson P, Dahlback B: Decreased plasma concentrations of apolipoprotein $M$ in sepsis and systemic inflammatory response syndromes. Crit Care 2012; 16: R60.

38. Christoffersen C, Nielsen LB: Apolipoprotein M - a new biomarker in sepsis. Crit Care 2012; 16: 126.

39. Jacobson JR, Garcia JG: Novel therapies for microvascular permeability in sepsis. Curr Drug Targets 2007; 8: 509514.

40. Camerer E, Regard JB, Cornelissen I, Srinivasan Y, Duong DN, Palmer D, Pham TH, Wong JS, Pappu R, Coughlin SR: Sphingosine-1-phosphate in the plasma compartment regulates basal and inflammation-induced vascular leak in mice. J Clin Invest 2009; 119: 1871-1879.

41. Christoffersen C, Obinata H, Kumaraswamy SB, Galvani S, Ahnstrom J, Sevvana M, Egerer-Sieber C, Muller YA, Hla T, Nielsen LB, Dahlback B: Endothelium-protective sphingosine-1-phosphate provided by HDL-associated apolipoprotein M. Proc Natl Acad Sci U S A 2011; 108: 9613-9618.

42. Zhao Y, Gorshkova IA, Berdyshev E, He D, Fu P, Ma W, Su Y, Usatyuk PV, Pendyala S, Oskouian B, Saba JD, Garcia JG, Natarajan V: Protection of LPS-induced murine acute lung injury by sphingosine-1-phosphate lyase suppression. Am J Respir Cell Mol Biol 2011; 45: 426-435.

43. Ebenezer DL, Fu P, Suryadevara V, Zhao Y, Natarajan V: Epigenetic regulation of pro-inflammatory cytokine secretion by sphingosine 1-phosphate (S1P) in acute lung injury: Role of S1P lyase. Adv Biol Regul 2017; 63: 156-166.

44. Hemdan NY, Weigel C, Reimann CM, Graler MH: Modulating sphingosine 1-phosphate signaling with DOP or FTY720 alleviates vascular and immune defects in mouse sepsis. Eur J Immunol 2016; 46: 2767-2777.

(Received, June 7, 2017)

(Accepted, November 24, 2017) 